
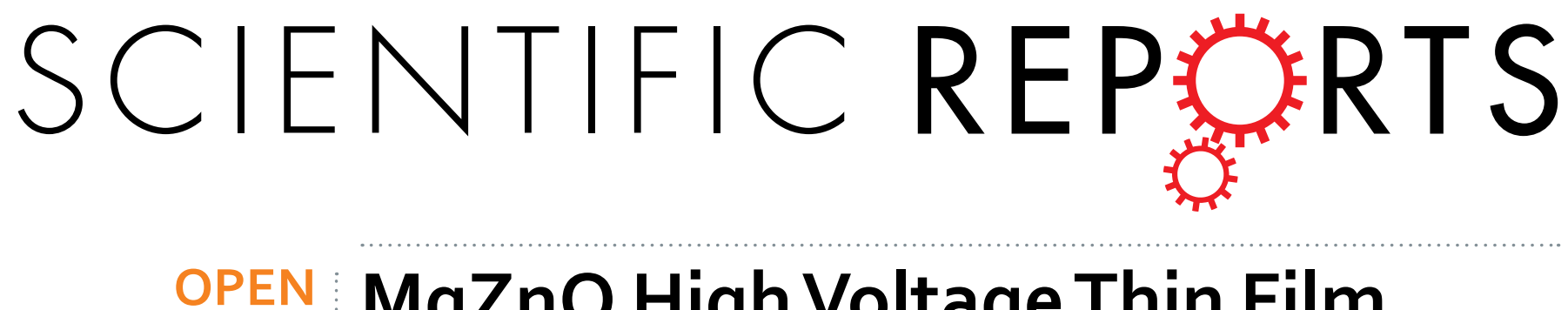

\title{
MgZnO High Voltage Thin Film Transistors on Glass for Inverters in Building Integrated Photovoltaics
}

Received: 17 June 2016

Accepted: 07 September 2016

Published: 10 October 2016

\section{Wen-Chiang Hong ${ }^{1}$, Chieh-Jen $\mathrm{Ku}^{1}$, Rui Li ${ }^{1}$, Siamak Abbaslou ${ }^{1}$, Pavel Reyes ${ }^{1}$, Szu-Ying Wang ${ }^{1}$, Guangyuan $\mathrm{Li}^{1}$, Ming $\mathrm{Lu}^{2}$, Kuang Sheng ${ }^{1,+}$ \& Yicheng $\mathrm{Lu}^{1}$}

Building integrated photovoltaics (BIPV) have attracted considerable interests because of its aesthetically attractive appearance and overall low cost. In BIPV, system integration on a glass substrate like windows is essential to cover a large area of a building with low cost. However, the conventional high voltage devices in inverters have to be built on the specially selected single crystal substrates, limiting its application for large area electronic systems, such as the BIPV. We demonstrate a Magnesium Zinc Oxide (MZO) based high voltage thin film transistor (HVTFT) built on a transparent glass substrate. The devices are designed with unique ring-type structures and use modulated $\mathrm{Mg}$ doping in the channel - gate dielectric interface, resulting in a blocking voltage of over $600 \mathrm{~V}$. In addition to BIPV, the MZO HVTFT based inverter technology also creates new opportunities for emerging selfpowered smart glass.

For decades, solar energy has been a leading technology in the search to replace fossil-fuel energy as a sustainable and clean energy source. However, cost and efficiency remain as major concerns ${ }^{1}$. As the price of photovoltaics (PV) modules has dropped, the inverters for energy conversion now count for more than $10 \%$ of the total cost of an entire PV system ${ }^{2}$. Additionally, in a conventional PV system all solar modules link to a central inverter; therefore, the overall system performance can be brought down by an underperforming module in the array, or by individual solar cells blocked from sunlight.

To solve this problem, and to optimize each individual solar panel, the micro-inverter technology ${ }^{3}$ has been proposed to embed inverters into each photovoltaic module. However, at the present time, the cost of micro-inverters is still higher than the cost of centralized inverters in small systems $(\leq 10 \mathrm{~kW})^{4}$. Another major factor hindering the wide adoption of solar energy is a conflict between aesthetics and energy saving. Many consumers find the placement of arrays of solar panels on buildings to be unsightly. Consequently, ways of integrating photovoltaics into buildings, i.e. Building Integrated Photovoltaics (BIPV) have been proposed ${ }^{5,6}$. BIPVs serve as building elements so that the appearance of houses won't be compromised due to the post-installation of solar panels.

To address these two major issues, we have explored the possibility of creating new inverters which can be integrated with solar modules to form a photovoltaic system on glass (PV-SOG). In a PV-SOG, in addition to inverters, all other components including solar cells and controller circuits can be designed and fabricated using the same process. Therefore, not only the cost but also the unit size of a PV system is reduced ${ }^{7}$. The transparency and scalability of the PV-SOG's exterior appearance make them attractive for application to BIPVs. Moreover, they possess better system reliability since every solar module is integrated with an individual inverter or inverter array. Our HVTFT inverter technology uses Magnesium Zinc Oxide (MZO) based wide band gap oxide semiconductor materials. Currently, the state-of-the-art high voltage and high power devices use the popular SiC and $\mathrm{GaN}$ transistors. However, both of these wide bandgap semiconductors require epitaxial growth at high temperature on strictly selected single-crystal substrates, which excludes their application in SOGs. In contrast, TFT technology made at low temperature has become a promising candidate for PV-SOGs. Several semiconductor materials have been tried out to make HVTFT devices. Amorphous Si and poly-Si HVTFTs have been studied since the $1980 \mathrm{~s}^{8-12}$. Martin et al. ${ }^{13}$ utilized offset gate structure in amorphous Si TFT to operate devices under $400 \mathrm{~V}$, and it

${ }^{1}$ Department of Electrical and Computer Engineering, Rutgers University, Piscataway, NJ 08854, USA. ${ }^{2}$ Center for Functional Nanomaterials, Brookhaven National Laboratory, Upton, New York 11973, USA. 'Present address: College of Electrical Engineering, Zhejiang University, Hangzhou 310027, China. Correspondence and requests for materials should be addressed toY.L. (email: ylu@rci.rutgers.edu) 
provided on/off ratio $\sim 10^{6}$. Chow et al. demonstrated amorphous Si TFTs, which can provide high voltage up to $800 \mathrm{~V}^{14}$; however, its poor performance (on/off ration $\sim 10^{4}$ ) limits its application. In the case of poly-Si HVTFTs, Cheng et al. ${ }^{15}$ demonstrated poly-Si lateral double diffused metal oxide semi-conductor (LDMOS). The blocking voltage reached $240 \mathrm{~V}$, and on/off ratio was $\sim 10^{6}$. Jamshidi-Roudbari ${ }^{16}$ et al. reported a multi-gate poly-Si HVTFTs which provided high current $(\sim 50 \mathrm{~mA})$ and high on/off ratio $\left(\sim 10^{8}\right)$ for actuator application. However, the bias condition $\left(\mathrm{V}_{\mathrm{GS}} \sim 30 \mathrm{~V}, \mathrm{~V}_{\mathrm{DS}} \sim 35 \mathrm{~V}\right)$ is not high enough for inverter application. Overall, poly-Si HVTFTs show better driving capability; however their low blocking voltage and non-uniformity from grain boundaries make them inadequate to meet the requirements of PV-SOG. In addition, Si-based TFT technology suffers from the absorption of visible light, restricting its application for transparent electronics. Organic TFTs which offer low cost and low process temperature have been used in display and RFID technologies. Recently, Smith et al. reported ${ }^{17}$ a high-voltage organic thin-film transistor (HVOTFT), which shows switch drain-to-source voltages higher than $300 \mathrm{~V}$ with a controlling voltage range from 0 to $20 \mathrm{~V}$. However, its low mobility, poor long-term stability, and the degradation at higher temperatures basically exclude its application in PV-SOGs, which operate under sunlight radiation, and its lifetime, like the regular residential solar cells, is expected to be more than 25 years.

Since Nomura et al. ${ }^{18}$ demonstrated Indium Gallium Zinc Oxide (IGZO) TFTs with high electrical performance at low process temperature, oxide semiconductor TFTs have emerged in many applications, especially in displays and transparent electronics. In the area of HVTFT, Jeong et al. ${ }^{19}$ reported an IGZO HVTFT, which operated at above $100 \mathrm{~V}$ with an on/off current ratio of $10^{7}$. Although this is beyond the regular operating voltage in regular TFTs, it is still not sufficient to be used in inverters for a solar PV system. Furthermore, it is desired to use indium-free materials due to the high cost of indium, especially in the case of large-area electronic systems such as solar cells. In addition, the toxicity of IGZO due to its high indium concentration is of considerable environmental concern.

In this work, we report a $\mathrm{Mg}_{0.03} \mathrm{Zn}_{0.97} \mathrm{O}(\mathrm{MZO})$ based HVTFT built on a transparent glass substrate. $\mathrm{ZnO}$ possesses a wide energy bandgap ( $\sim 3.34 \mathrm{eV}$ at room temperature), similar to SiC and GaN. As a TFT channel material, $\mathrm{ZnO}$ can sustain a high electrical field ${ }^{20,21}$, which is particularly important for high voltage devices, such as inverters. The thermal conductivity of $\mathrm{ZnO}$ is as good as $\mathrm{Si}^{22} . \mathrm{ZnO}$ and $\mathrm{MZO}$ have been used for various solar cells ${ }^{23,24}$; thus, MZO inverters would benefit PV-SOG technology as various components including cells, control devices, and inverters can be integrated on the same glass substrate. However, TFTs using pure $\mathrm{ZnO}$ as a channel suffer from threshold voltage shift and thermal instability ${ }^{25,26}$, which would hinder its use as an inverter. To keep intrinsic advantages but resolve the instability accompanied with pure $\mathrm{ZnO}$, we have introduced a small amount of Magnesium ( $\mathrm{Mg}$ ) into $\mathrm{ZnO}$ to form a ternary $\mathrm{Mg}_{\mathrm{x}} \mathrm{Zn}_{1-\mathrm{x}} \mathrm{O}(\mathrm{MZO})$ as the TFT channel material which suppresses oxygen vacancy in the channel layer. In addition, the interface between the gate dielectric $\left(\mathrm{SiO}_{2}\right)$ and the MZO channel is specially designed by modulation-doping Mg into MZO to significantly increase the TFT blocking voltage. This MZO based HVTFT on glass technology naturally fits the PV-SOGs, which are not only suitable for BIPV but also promising for the self-powered smart glasses ${ }^{27}$. It may also create opportunities for other high voltage applications, such as high dc voltage sensing ${ }^{28}$, high-speed printers ${ }^{29}$, flat-panel x-ray imaging systems for medical radiology $\mathrm{y}^{30}$, and space engineering ${ }^{31}$.

\section{Results}

Device structure. The regular TFT has a rectangular channel. For an HVTFT, such a design would introduce non-uniform electrical field distribution with the highest field located at the corners of the channel, which limits the blocking voltage of the devices. To solve this problem, a ring structure is designed as shown in Fig. 1. The electrical field distributions of the ring and the rectangular structures are included in the supporting information. From the comparison between these two configurations, it can be seen that the field distribution from drain to source in the ring structure is much more uniform and the highest field is $\sim 50 \%$ less than in the rectangular counterpart. The HVTFT has a bottom gate inverted-staggered configuration, and it includes two offset regions: gate to drain and gate to source, respectively. For comparisons, three types of materials were deposited on a SiO $\mathrm{Sie}_{2}$ lectric layer, serving as the channel layer with a thickness of $50 \mathrm{~nm}$ : (i) pure $\mathrm{ZnO}$, (ii) $\mathrm{Mg}_{0.03} \mathrm{Zn}_{0.97} \mathrm{O}$ (MZO), and (iii) $\mathrm{Mg}_{0.03} \mathrm{Zn}_{0.97} \mathrm{O}$ plus a modulation-doped thin layer (m-MZO). In the m-MZO HVTFT, a modulation-doped $10 \mathrm{~nm} \mathrm{Mg} \mathrm{Zn}_{1-\mathrm{y}} \mathrm{O}$ transition layer (MZO-TL) is inserted between the $\mathrm{MZO}$ channel layer and the $\mathrm{SiO}_{2}$ dielectric layer, and the $\mathrm{Mg}$ composition (y) in the $\mathrm{Mg}_{\mathrm{y}} \mathrm{Zn}_{1-\mathrm{y}} \mathrm{O}$ TL decreases from the side adjacent to $\mathrm{SiO}_{2}(\mathrm{y}=1)$ to the other side adjacent to the channel $(y=0.03)$.

Transfer characteristics and thermal stability. The transfer characteristics of HVTFTs with three different channel materials and structures are shown in Fig. 2a. Compared with ZnO HVTFT, MZO HVTFT shows a better subthreshold slope (S.S.) and on-current. The most significant improvement of the MZO over the pure $\mathrm{ZnO}$ channel layer is in its thermal stability, which is one of the critical requirements for high voltage devices being integrated into PV-SOGs. By measuring the transfer characteristics of HVTFTs at different temperatures, the shifts of threshold voltage $\left(\Delta \mathrm{V}_{\mathrm{th}}\right)$ are compared in Fig. $2 \mathrm{~b}$, and the detail definition of the shifts of threshold voltage can be found in the Supplementary Information. As temperature increases from $294 \mathrm{~K}$ (room temperature) to $367 \mathrm{~K}$, MZO HVTFT only shows a threshold voltage shift $\Delta \mathrm{V}_{\text {th }}$ of $-6 \mathrm{~V}$ in comparison to $\Delta \mathrm{V}_{\text {th }}$ of $-10.5 \mathrm{~V}$ in the pure $\mathrm{ZnO}$ counterpart. This negative shift of threshold voltage at higher temperatures results from the thermally activated electrons from the trap states located in the channel and in the interface between the channel and gate dielectric $\mathrm{SiO}_{2}$.

In Fig. 2c, the activation energy of drain currents at different gate biases is extracted from the Arrhenius plot $\left(\ln I_{D}\right.$ vs. $\left.T^{-1}\right)$ in equation (1):

$$
I_{D}=I_{D 0} e^{-E_{a} / k_{B} T}
$$


a

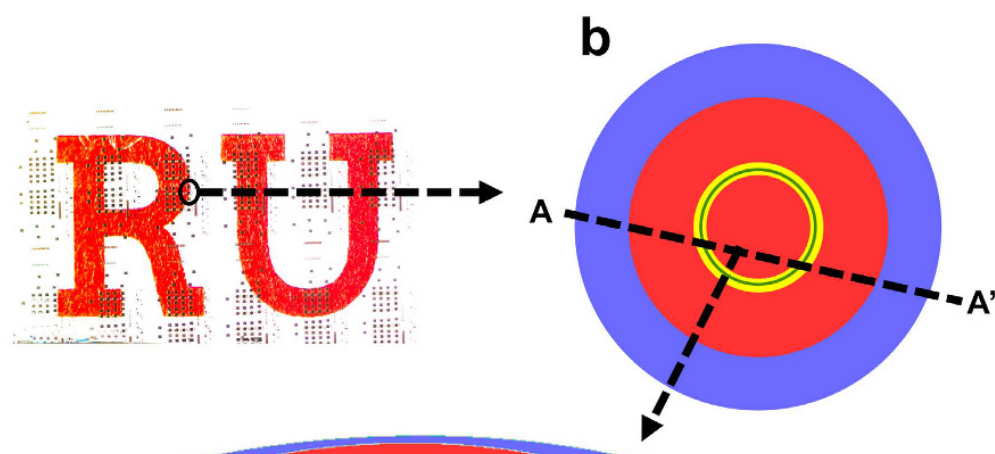

C
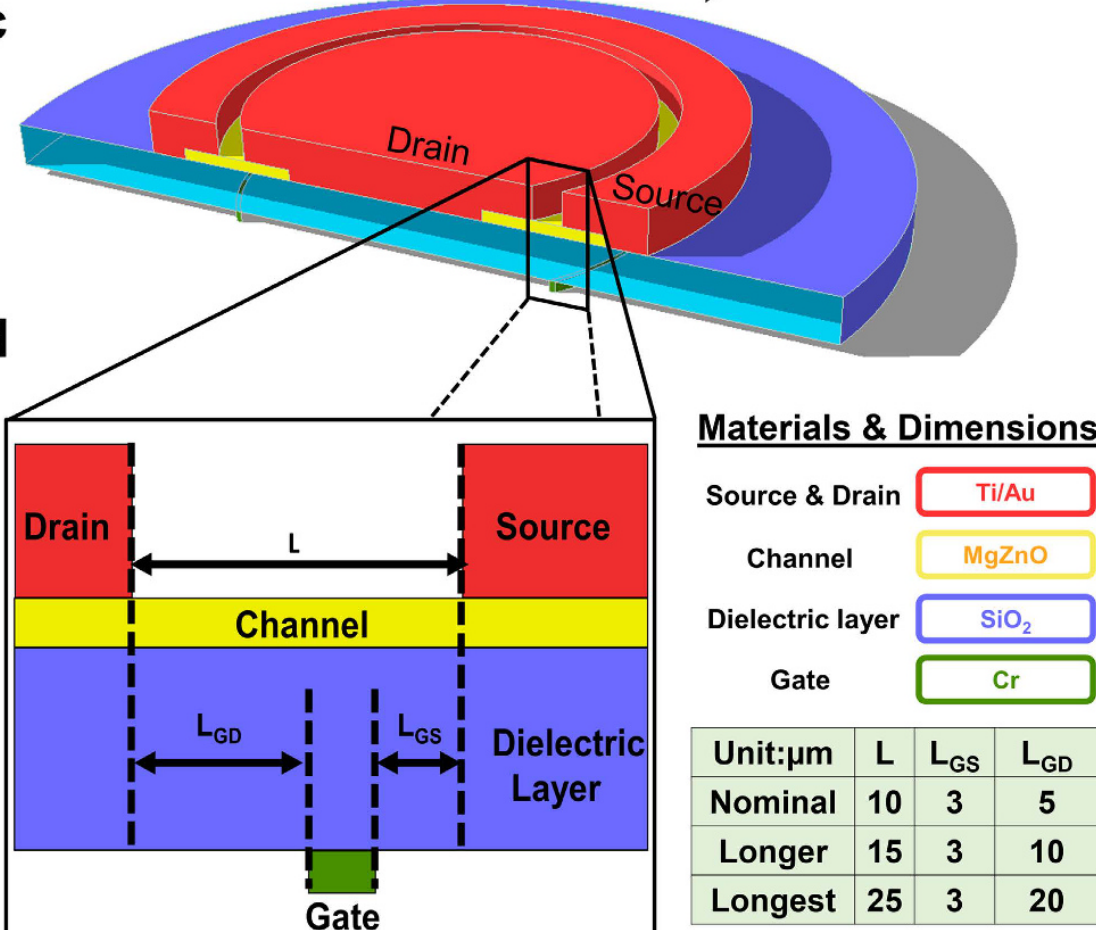

Figure 1. The overview of the structures of HVTFTs. (a) A photograph displays the HVTFTs on a transparent glass substrate. Schematic diagrams of an MZO HVTFT with a ring structure are shown: (b) the top view and (c) the three-dimensional cross-sectional structure along A-A' in (b,d) the dimensions and structure of a single HVTFT device.

where $I_{D 0}$ is the drain current constant, $k_{B}$ the Boltzmann constant, $T$ the temperature in Kelvin, and $E_{a}$ the activation energy of drain current.

If the trap density in TFT is high, the moving rate of the Fermi level with respect to the gate bias from the deep level to the conduction band is roughly inversely proportional to the total trap density. The steeper falling rate $(0.177 \mathrm{eV} / \mathrm{V})$ of the MZO HVTFT, as opposed to that of the ZnO HVTFT $(0.107 \mathrm{eV} / \mathrm{V})$, indicates that the MZO TFT has a lower trap density. Because both MZO and ZnO HVTFTs are fabricated on the same $\mathrm{SiO}_{2}$ gate dielectric layer, the interface trap densities of the two devices are similar. Therefore, the improvement of thermal stability in MZO HVTFT over ZnO HVTFT is mainly attributed to the reduction of traps in the bulk channel. In fact, the $\mathrm{Mg}-\mathrm{O}$ has a stronger bonding than $\mathrm{Zn}-\mathrm{O}^{25}$, resulting in the lower density of oxygen vacancy in the $\mathrm{MZO}$ channel than that of the pure $\mathrm{ZnO}$ channel.

In the comparison between MZO HVTFT and m-MZO HVTFT, it is found that the later shows an order higher on-current and a steeper S.S. than the MZO counterpart. The steepest falling rate $(0.246 \mathrm{eV} / \mathrm{V})$ of the activation energy of the drain current suggests a nearly $40 \%$ lower total trap density in m-MZO HVTFT than in MZO HVTFT. Since both of MZO and m-MZO HVTFTs are made up of the same MZO channel and $\mathrm{SiO}_{2}$ gate dielectric layer, these differences in characteristics between the two HVTFTs are mainly caused by the different interface properties between the channel and gate dielectric layer. Specifically, the unique interface design and engineering using modulation doping of $\mathrm{Mg}$ in $\mathrm{m}$-MZO HVTFT reduces the interface trap density. Therefore, the total trap density in m-MZO HVTFT is lower than in the MZO HVTFT.

$\mathrm{Mg}_{\mathrm{y}} \mathrm{Zn}_{1-\mathrm{y}} \mathrm{O}$ transition layer (MZO-TL) as a diffusion barrier. To understand the effect of the MZO-TL on improving the properties of the interface between the channel and gate dielectric layer, the cross sections of 
a

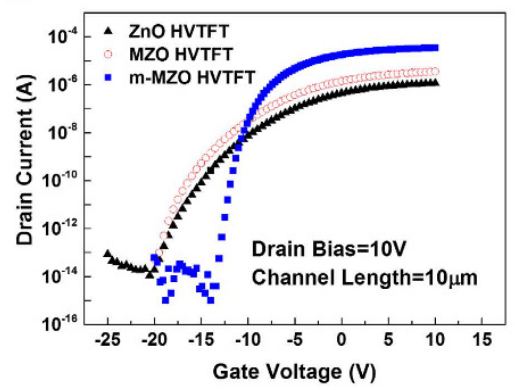

b

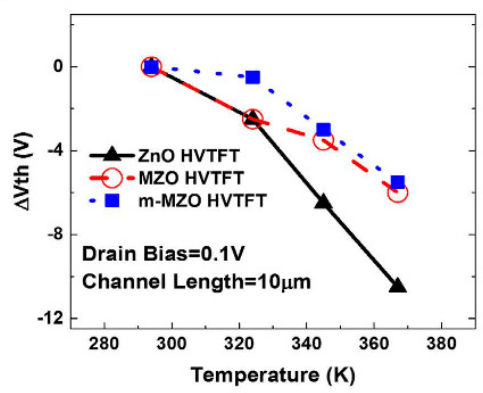

C

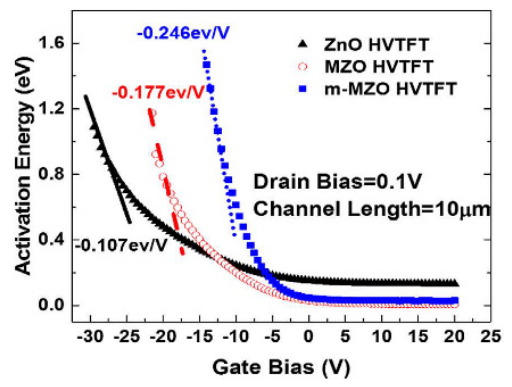

Figure 2. The electrical performances of HVTFTs. (a) Transfer characteristics of $\mathrm{ZnO}, \mathrm{MZO}$ and $\mathrm{m}-\mathrm{MZO}$ HVTFTs with $V_{D S}=10 \mathrm{~V}$. (b) The shift of threshold voltage of HVTFTs at different temperatures. (detail definition of the shifts of threshold voltage can be found in the Supplementary Information.) (c) The extracted activation energy of drain current as a function of gate bias in HVTFTs.

a

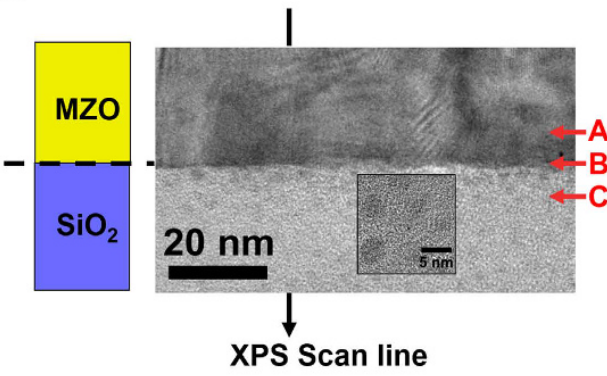

$\mathrm{SiO}_{2} / \mathrm{MZO}$ interface in MZO HVTFT

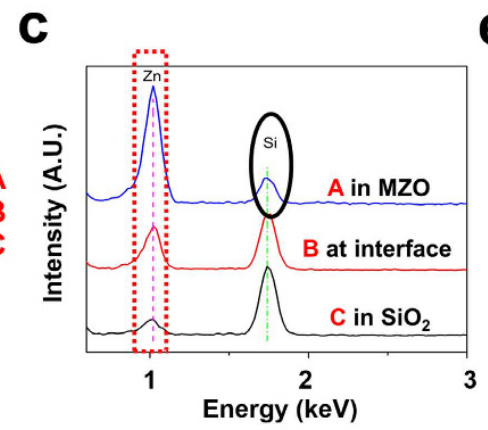

e

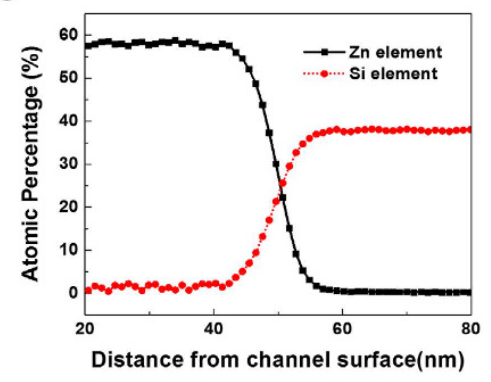

\section{$\mathrm{SiO}_{2}$ /Transition Layer/MZO interface in m-MZO HVTFT}

b

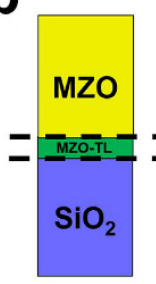

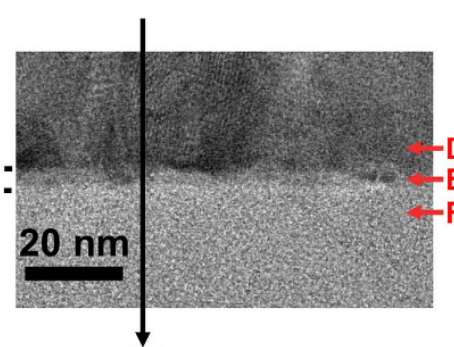

XPS Scan line
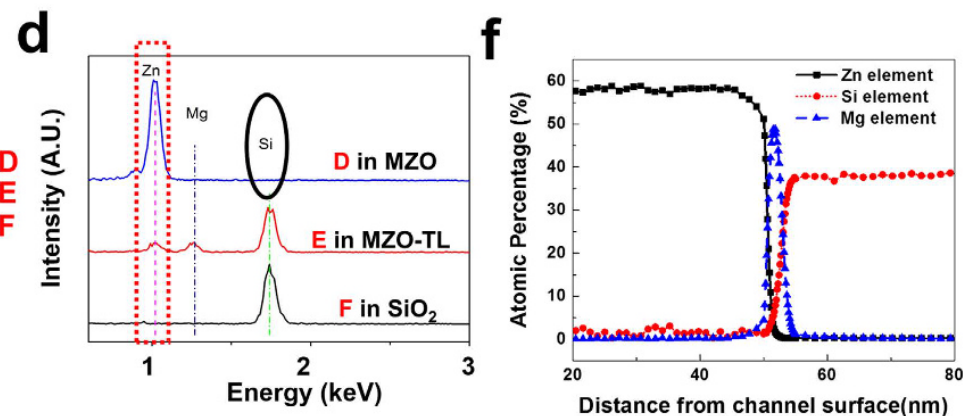

Figure 3. Material characterization near the interfaces. TEM images of the interfaces between the channel layer and the $\mathrm{SiO}_{2}$ dielectric layer (a) without a transition layer (for MZO HVTFT) and (b) with a modulation doped transition layer (for m-MZO HVTFT). The inset of Fig. 3(a) features the gray dots found in the $\mathrm{SiO}_{2}$ layer in the MZO sample. EDS spectra of $\mathrm{Zn}, \mathrm{Mg}$ and Si elements at the different locations (marked in the TEM images) across the channel - gate dielectric interface for (c) MZO and (d) m-MZO samples. Depth profiles of atomic percentage of $\mathrm{Si}, \mathrm{Zn}$, and $\mathrm{Mg}$ from XPS measurements of (e) MZO and (f) $\mathrm{m}$-MZO samples along the scan lines shown in the TEM pictures. The oxygen profile is not included.

HVTFTs prepared by a Focus Ion Beam (FIB) were studied using a transmission electron microscopy (TEM). Figure 3a and bshow the images of the interface regions in MZO and m-MZO HVTFT samples, respectively. As shown in the inset of Fig. 3a, the gray dots are observed only in the $\mathrm{SiO}_{2}$ dielectric layer of the MZO HVTFT sample. To identify these dots, energy-dispersive X-ray spectroscopy (EDS) was used to analyze the elemental composition of the films at different positions (point "A" to "F") across the interface. As shown in the dashed box of Fig. 3c, the Zn peak in MZO HVTFT appears not only in the MZO channel area (point A) but also near the interface (point $\mathrm{B}$ ) and even inside the gate dielectric $\mathrm{SiO}_{2}$ layer (point $\mathrm{C}$ ). The EDS results further confirm that the observed gray dots are related to the $\mathrm{Zn}$ element. Moreover, in the EDS spectrum, there is a Si peak marked 
a

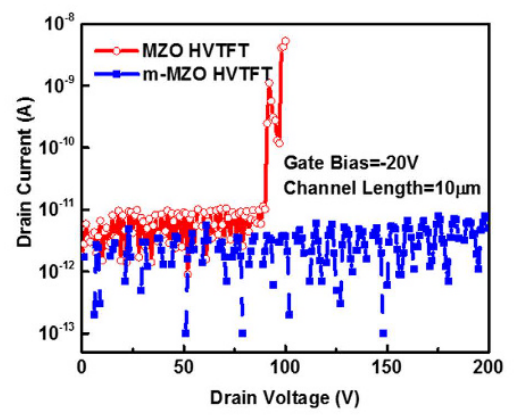

b

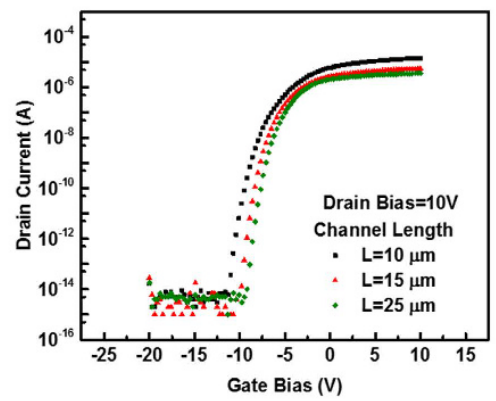

C

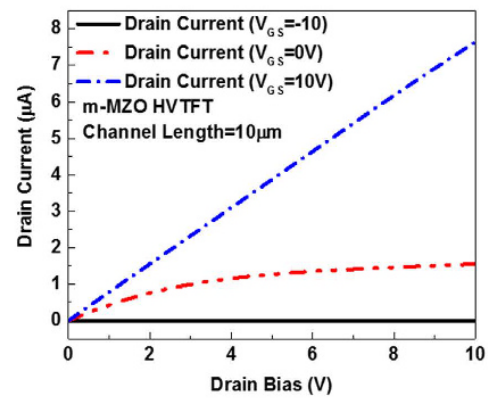

d

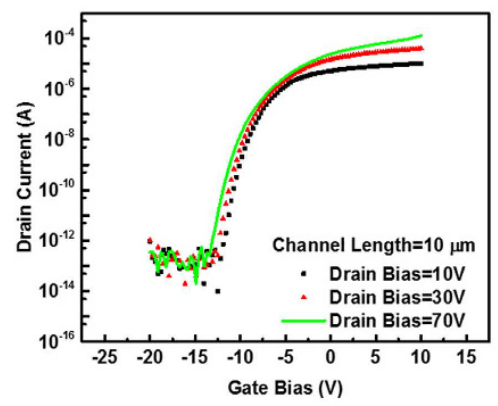

e

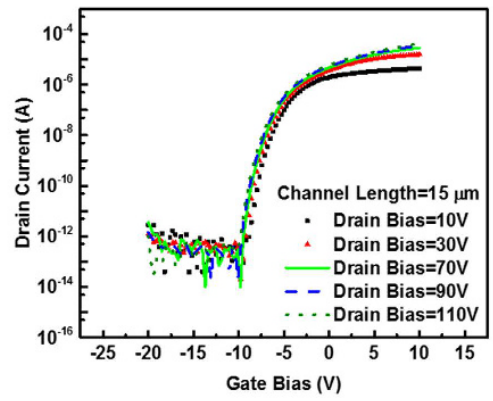

f

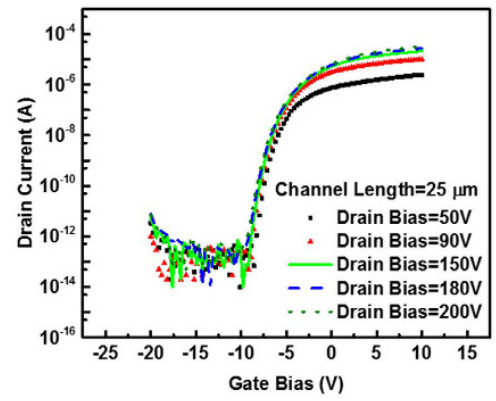

Figure 4. The electrical performances at high bias conditions and the blocking capabilities of HVTFTs: (a) drain leakage current of off state in MZO and m-MZO HVTFTs; (b) transfer characteristics of m-MZO HVTFTs with different channel lengths (the detailed dimensions of m-MZO HVTFTs are listed in the Table of Fig. 1d.); (c) the output characteristics of $\mathrm{m}-\mathrm{MZO}$ HVTFT with a channel length $\mathrm{L}=10 \mu \mathrm{m}$; (d-f) the transfer characteristics at high bias conditions of $\mathrm{m}-\mathrm{MZO}$ HVTFTs with a channel length $\mathrm{L}=10,15$, and $25 \mu \mathrm{m}$, respectively.

by the "circle" in the MZO area (point A), The results indicate that the interdiffusions occur across the interface (point B): $\mathrm{Zn}$ diffuses from the $\mathrm{MZO}$ layer into $\mathrm{SiO}_{2}$ while $\mathrm{Si}$ diffuses from the $\mathrm{SiO}_{2}$ layer into the $\mathrm{MZO}$ layer. In contrast, for m-MZO HVTFT, as shown in the dashed box of Fig. 3d, the Zn peak appears in the MZO area (point $\mathrm{D}$ ), only a tiny peak appears near the interface (point $\mathrm{E}$ ), but no $\mathrm{Zn}$ peak is observed inside the $\mathrm{SiO}_{2}$ layer (point F). On the other hand, as shown in the circle, there is no Si peak detected in the MZO area (point D). Therefore, the modulation-doped thin transition layer inserted between the $\mathrm{MZO}$ and $\mathrm{SiO}_{2}$ acts as a diffusion barrier, which hinders $\mathrm{Zn}$ and $\mathrm{Si}$ diffusion across the interface between the channel and gate dielectric layer.

$\mathrm{X}$-ray photoelectron spectroscopy (XPS) was used to estimate the atomic percentages of different elements in the interface regions. The depth profiles were obtained by using the in-situ sputtering process. Figure $3 \mathrm{e}, \mathrm{f}$ show depth profiles of atomic percentages of $\mathrm{Si}, \mathrm{Zn}$, and $\mathrm{Mg}$ in the MZO and the m-MZO HVTFT, respectively. A small amount (3\%) of Mg doping is barely shown inside the MZO channels in both of MZO and $\mathrm{m}-\mathrm{MZO}$ HVTFT samples due to the detection limit of XPS. However, a narrow peak of $\mathrm{Mg}$ does appear in the $\mathrm{m}-\mathrm{MZO}$ HVTFT, produced from the $\mathrm{Mg}_{\mathrm{y}} \mathrm{Zn}_{1-\mathrm{y}} \mathrm{O}$ transition layer (MZO-TL). In the MZO sample, $\mathrm{Zn}$ diffuses extensively into the $\mathrm{SiO}_{2}$ layer as indicated by the length of overlapped XPS profiles of $\mathrm{SiO}_{2}$ and $\mathrm{MZO}$. In contrast, there is an abrupt interface with negligible overlapped profiles of $\mathrm{Si}$ and $\mathrm{Zn}$ in the $\mathrm{m}$-MZO sample. TEM/EDS and XPS characterizations provide consistent results that the phenomenal interdiffusion between the $\mathrm{SiO}_{2}$ gate dielectric layer and the MZO channel layer is only detected in the MZO HVTFT. The MZO-TL in m-MZO HVTFT acts as a diffusion barrier, which effectively blocks the interdiffusion of $\mathrm{Zn}$ from the $\mathrm{MZO}$ to $\mathrm{SiO}_{2}$, as well as $\mathrm{Si}_{\text {from }} \mathrm{SiO}_{2}$ to the MZO channel.

High voltage blocking capability. Having the capability of blocking high voltages and operating at high bias conditions reliably are the essential characteristics for the application of high voltage transistors in PV inverters. Since ZnO HVTFT fails to show thermal stability, we here only compare MZO and m-MZO HVTFTs. The results of blocking voltages of MZO and m-MZO HVTFTs with the same channel length $(\mathrm{L}=10 \mu \mathrm{m})$ are shown in Fig. 4a. The drain leakage current of MZO HVTFT increases abruptly, and the device burns down at $\mathrm{V}_{\mathrm{DS}}=90 \mathrm{~V}$. In contrast, the drain leakage current of m-MZO HVTFT keeps as low as $10^{-12} \mathrm{~A}$ even at much higher $\mathrm{V}_{\mathrm{DS}}=200 \mathrm{~V}$ (here $200 \mathrm{~V}$ is the limitation of HP-4156C used for testing).

A comparison of the transfer characteristics at normal bias (drain bias $=10 \mathrm{~V}$ ) among three $\mathrm{m}-\mathrm{MZO}$ HVTFTs with different channel lengths is presented in Fig. 4b. There is a trade-off between blocking capability and driving capability in m-MZO HVTFT. As the channel length increases, the blocking voltage increases; however, the on-current drops. The values of the blocking voltage/on-current for the nominal $(\mathrm{L}=10 \mu \mathrm{m})$, longer $(\mathrm{L}=15 \mu \mathrm{m})$, 


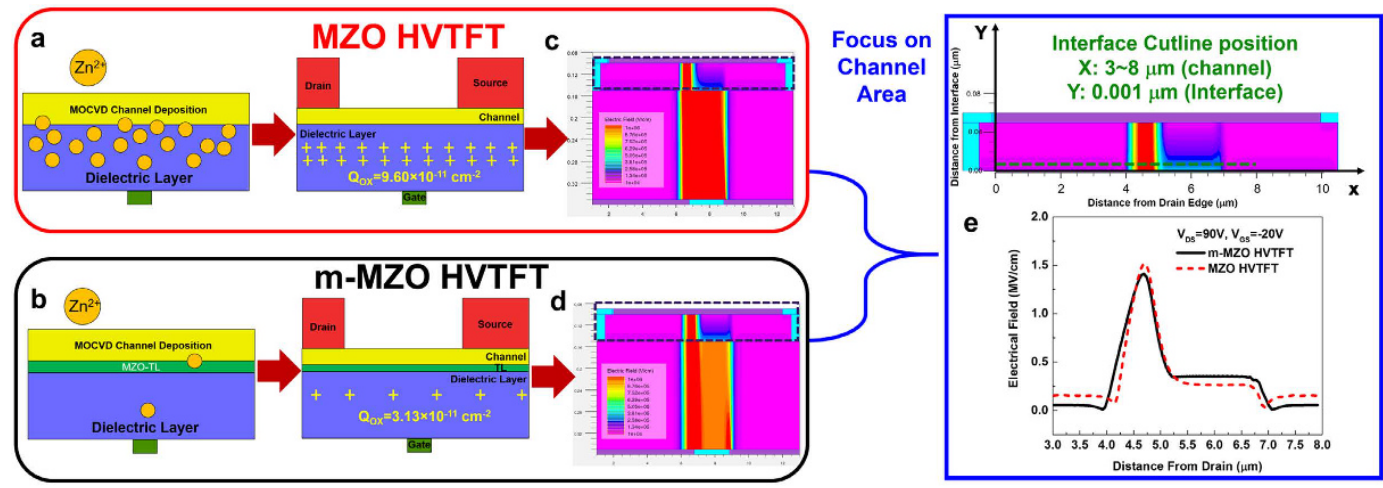

Figure 5. The simulation results of electrical field distribution in HVTFTs. Schematic diagrams of different interface designs and layer structures of (a) MZO and (b) m-MZO HVTFT, respectively. The SILVACO simulation results on two-dimensional distributions of the critical electrical field in the nominal $(\mathrm{L}=10 \mu \mathrm{m})$ channels of (c) MZO HVTFT and (d) m-MZO HVTFT, respectively. (e) A comparison of the electrical field of MZO HVTFT and m-MZO HVTFT. The cutline of the electrical field is near the interface of channel/gate dielectric and above the gate edge where the maximum electrical field locates.

and longest $(\mathrm{L}=25 \mu \mathrm{m}) \mathrm{m}$-MZO HVTFT are $300 \mathrm{~V} / 3.5 \times 10^{-5} \mathrm{~A}, 427 \mathrm{~V} / 6.61 \times 10^{-6} \mathrm{~A}$, and $609 \mathrm{~V} / 4.57 \times 10^{-6} \mathrm{~A}$, respectively. The output characteristics of $\mathrm{m}-\mathrm{MZO}$ HVTFT of $10 \mu \mathrm{m}$ are presented in Fig. $4 \mathrm{c}$. It shows better saturation behavior at low gate bias. At high gate bias, the drain current increases as the drain bias increases. This kink effect was also observed in the IGZO HVTFT ${ }^{19}$. It might be related to the channel length modulation induced self-heating effect ${ }^{19,32}$.

The m-MZO HVTFTs with channel length of 10,15 , and $25 \mu \mathrm{m}$ have the highest operating drain bias of 70 , 110 , and $200 \mathrm{~V}$ as shown in Fig. 4d-f, respectively. The maximum drain voltage without degrading the on-current defines the highest operating drain bias of each HVTFT, except for the case of $25 \mu \mathrm{m}$ which only shows $200 \mathrm{~V}$ due to the limitation of the testing equipment. Under these high drain bias conditions, all m-MZO HVTFTs show a high on/off ratio of more than $10^{7}$, and the $\mathrm{V}_{\mathrm{OFF}}$ and off-current almost constant at any drain bias condition. Theses features indicate that $\mathrm{m}-\mathrm{MZO}$ HVTFTs are stable even under high bias conditions. The $\mathrm{m}-\mathrm{MZO}$ HVTFT of $25 \mu \mathrm{m}$ can operate at drain bias of $200 \mathrm{~V}$ with a blocking capability over $600 \mathrm{~V}$, suitable to be used as an inverter of PV-SOG.

\section{Discussion}

The interface engineering using a modulation-doped thin MZO transition layer (MZO-TL) in the m-MZO HVTFT improves transfer characteristics, thus enables high voltage blocking capability. Such improvements are mainly attributed to the prevention of $\mathrm{Zn}$ diffusion into the $\mathrm{SiO}_{2}$ dielectric layer. $\mathrm{Zn}$ could diffuse as ions, such as $\mathrm{Zn}^{2+}$ into the dielectric layer and then become the fixed charges in $\mathrm{SiO}_{2}$. Because the positive $\mathrm{Zn}^{2+}$ trapped inside $\mathrm{SiO}_{2}$ would attract electrons, it requires an extra negative gate bias voltage to deplete the channel. As a result, MZO HVTFT, which has extra diffusion of $\mathrm{Zn}^{2+}$ into $\mathrm{SiO}_{2}$, has more negative $\mathrm{V}_{\mathrm{OFF}}$ than m-MZO HVTFT does. Moreover, the out-diffusion of $\mathrm{Zn}$ from the $\mathrm{MZO}$ channel layer would generate $\mathrm{Zn}$-related defects, such as $\mathrm{Zn}$ vacancies and $\mathrm{Zn}$ interstitials in the $\mathrm{MZO}$ channel layer, especially near the $\mathrm{MZO} / \mathrm{SiO}_{2}$ interface, thus degrading the electrical performance of transistors. The total trap density from the subthreshold slope (S.S.) can be estimated in the equation $(2)^{33}$ :

$$
S . S=\log _{e} 10 \times \frac{k_{B} T}{q}\left(1+q \frac{t N_{b u l k}+D_{i t}}{C_{G}}\right)
$$

where $q$ is the elementary electric charge, $k_{B}$ the Boltzmann constant, $T$ the temperature in Kelvin, $t$ the channel thickness, $N_{b u l k}$ the bulk trap density, $D_{i t}$ the interface trap density, and $C_{G}$ the capacitance per area of the gate dielectric layer $\left(C_{G}\right.$ is $1.73 \times 10^{-4} \mathrm{~F} / \mathrm{m}^{2}$ and $1.69 \times 10^{-4} \mathrm{~F} / \mathrm{m}^{2}$ for MZO and m-MZO HVTFT, respectively. The theoretical values of $\mathrm{SiO}_{2}=3.9$ and $\mathrm{MgO}=9.90^{34}$ are used in estimation). The total trap density includes the bulk trap $\left(t N_{b u l k}\right)$ and the interface trap $\left(D_{i t}\right)$, which are calculated to be $2.14 \times 10^{12} \mathrm{~cm}^{-2}$ and $8.36 \times 10^{11} \mathrm{~cm}^{-2}$ for MZO and $\mathrm{m}-\mathrm{MZO}$ HVTFT, respectively. Since the channel material is the same, the difference in the total charge density between HVTFT and m-HVTFT is approximately equal to the reduction of the interface trap density, which is $1.3 \times 10^{12} \mathrm{~cm}^{-2}$. By adding a modulation doped transition layer into the $\mathrm{m}$-MZO HVTFT, Zn diffusion into the $\mathrm{SiO}_{2}$ dielectric layer is significantly suppressed. The interface engineering successfully adjusts the threshold voltage $\mathrm{V}_{\text {th }}$ close to $0 \mathrm{~V}$ and makes the S.S. steeper than that of MZO HVTFT.

The addition of the MZO-TL also enables the higher blocking voltage. The ideal breakdown field of a pure $\mathrm{MgO}$ is $12 \mathrm{MV} / \mathrm{cm}^{35}$. Although the effective thickness of the dielectric layer increases by adding the MZO-TL, a $10 \mathrm{~nm}$ MZO transition layer can not provide more than $12 \mathrm{~V}$ of the blocking voltage. Furthermore, the gate leakage current keeps at a similar level in both HVTFTs after the breakdown at high drain bias. Therefore, the significant enhancement of the blocking voltage in m-MZO HVTFT cannot be attributed to the extra voltage drop on the MZO-TL. In order to understand the fundamental cause of the improvement in blocking voltage, SILVACO software was used to simulate the electric field distributions in the two different devices. As drawn in the Fig. 5a, 
MZO HVTFT possesses extra positive oxide charges in comparison to m-MZO HVTFT. The amount of the extra equivalent oxide charges per unit area $\left(\mathrm{Q}_{\mathrm{OX}}\right)$ is estimated based on the equation (3):

$$
Q_{O X}=-\Delta V_{\text {OFF }} \times C_{G}
$$

where $\mathrm{C}_{\mathrm{G}}$ is the capacitance per area of the gate dielectric layer. The difference in $\mathrm{V}_{\mathrm{OFF}}$ between $\mathrm{MZO}$ and $\mathrm{m}-\mathrm{MZO}$ HVTFT is around $-6 \mathrm{~V}$, so the extra $6.47 \times 10^{11} \mathrm{~cm}^{-2}$ of positive charges are placed in the MZO HVTFT. The impact of oxide charges near the interface on electrical field distribution in the device is shown in Fig. 5 . The more detailed simulation, including the consideration of the gate connection between the gate ring and the probing pad can be found in Supplementary Information. The maximum values of the electrical field near the interface are $1,520 \mathrm{kV} / \mathrm{cm}$ and $1,410 \mathrm{kV} / \mathrm{cm}$ for MZO and m-MZO HVTFT, respectively. The reduction of the maximum electrical field allows m-MZO HVTFT to operate at higher drain bias, enabling higher blocking voltage. Overall, the MZO-TL in m-MZO HVTFT acts as a barrier against $\mathrm{Zn}$ diffusion so that the interface states and trapped charges are reduced. This improvement leads to the decrease of the maximum electrical field near the channel-gate dielectric interface, resulting in the increase of the blocking voltage.

In summary, we have demonstrated the MZO high voltage thin film transistors on a transparent glass substrate. The ring structure design reduces the electric field crowding effect in the devices. The thermal stability is enhanced by doping $\mathrm{ZnO}$ with a small amount of $\mathrm{Mg}$ to form the $\mathrm{MZO}$ channel layer. The interface design and engineering are conducted by inserting a modulation-doped ultra-thin MZO transition layer between the $\mathrm{SiO}_{2}$ gate dielectric layer and the MZO channel, and they significantly improve the subthreshold slope and on-current values. More importantly, it enables high blocking voltage of $609 \mathrm{~V}$ with an on/off ratio of $3.3 \times 10^{8}$, and operating voltage over $200 \mathrm{~V}$. The comprehensive characterizations confirm that the enhancement in the HVTFT performance is mainly attributed to the reduction in interface trap density and trapped charges, which leads to the reduction of the maximum electric field in the channel. This MZO-based HVTFT on glass technology is promising to serve as the solar inverter in PV-SOG technology to implement the emerging BIPV and self-powered smart glass

\section{Methods}

Material Preparation and Device Fabrication Process. The HVTFTs were fabricated on $0.4 \mathrm{~mm}$ thick commercial glass substrates. A $50 \mathrm{~nm}$ chromium (Cr) layer was deposited by sputtering, and patterned using a dry etching process to serve as the bottom gate electrode. Then, a $200 \mathrm{~nm} \mathrm{SiO}$ layer was deposited by plasma enhanced chemical vapor deposition (PECVD) as the gate dielectric layer. Following the $\mathrm{SiO}_{2}$ deposition, the channel was deposited using metal organic chemical vapor deposition (MOCVD) at $400{ }^{\circ} \mathrm{C}$. DeZn (diethyl zinc) and MCp2Mg (bis (methylcyclopentadienyl) magnesium) were used as the precursors for $\mathrm{Zn}$ and $\mathrm{Mg}$, respectively. Three types of channel layers (thickness of $50 \mathrm{~nm}$ ) were deposited on $\mathrm{SiO}_{2}$ : (i) pure $\mathrm{ZnO}$, (ii) $\mathrm{Mg}_{0.03} \mathrm{Zn}_{0.97} \mathrm{O}(\mathrm{MZO})$, and (iii) modulation-doped $\mathrm{Mg}_{0.03} \mathrm{Zn}_{0.97} \mathrm{O}$ (m-MZO). In the m-MZO HVTFT, a $10 \mathrm{~nm}$ modulation-doped $\mathrm{Mg}_{\mathrm{y}} \mathrm{Zn}_{1-\mathrm{y}} \mathrm{O}$ transition layer (MZO-TL) was inserted between the $\mathrm{Mg}_{0.03} \mathrm{Zn}_{0.97} \mathrm{O}$ (MZO) channel layer and the $\mathrm{SiO}_{2}$ dielectric layer, and the $\mathrm{Mg}$ composition (y) in the TL decreases from the side adjacent to $\mathrm{SiO}_{2}(\mathrm{y}=1)$ to the other side adjacent to the channel $(\mathrm{y}=0.03)$. The source and drain metallization (100 nm titanium $(\mathrm{Ti}) / 50 \mathrm{~nm}$ gold $(\mathrm{Au})$ ) was deposited using electron beam evaporation, followed by a normal lift-off process. A photoresist film was coated on top of the TFT channel, serving as a passivation layer to prevent ambient absorption/desorption during electrical testing. HVTFTs with three different channel lengths are listed in the table of Fig. 1d. The channel lengths/gate-to-drain offset lengths are $10 / 5 \mu \mathrm{m}$, $15 / 10 \mu \mathrm{m}$, and $25 / 20 \mu \mathrm{m}$ for nominal, longer, and longest HVTFT, respectively. The gate-to-source offset is kept the same of $3 \mu \mathrm{m}$.

Testing Conditions. The electrical measurements under the low bias were conducted using an HP-4156C with an HP-41501B Pulse Generator. With the boost from the connection of a pulse generator, the maximum voltage of the HP-4156C electrical testing system was limited to be $200 \mathrm{~V}$. The system which had a current resolution of $1 \times 10^{-15} \mathrm{~A}$ was used for all transfer characteristics. For electrical measurements under high bias, a high voltage testing system was built based on a Tektronix 370 with the probe station. As the current resolution of Tektronix 370 only reached $1 \times 10^{-6} \mathrm{~A}$, it was only used for the testing of blocking voltages. In order to avoid problems with arcing and tracking due to environmental conditions, the devices were immersed in Fluorinert FC-40 during the high voltage measurements. The electrical measurements at different temperatures were conducted using an Agilent 1500B. All measurements were conducted in a light-tight probe station.

The samples used in the material analysis were prepared under the same device process except that the materials samples did not go through patterning and metallization processes. The structural and interfacial properties were analyzed using JEOL 2100F Field Emission Lorentz Transmission Electron Microscopy (TEM), Energy-dispersive X-ray spectroscopy (EDS), and X-ray photoelectron spectroscopy (XPS). The TEM samples were prepared by using a FEI HELIOS 600 Dual Beam Focus Ion Beam (FIB).

\section{References}

1. Mason, N. Manufacturing technology: Fabrication innovations. Nat. Photonics 2, 281-283 (2008).

2. Semiconductor Equipment and Materials International (SEMI). International Technology Roadmap for Photovoltaic 2014 Results Revision 1. Itrpv 1-38. Available at: http://www.itrpv.net/Reports/Downloads/ (2015).

3. Sher, H. A. \& Addoweesh, K. E. Micro-inverters - Promising solutions in solar photovoltaics. Energy Sustain. Dev. 16, 389-400 (2012).

4. Barbose, G. Tracking the Sun VI: An Historical Summary of the Installed Price of Photovoltaics in the United States from 1998 to 2012. Lawrence Berkeley National Laboratory. Available at: http://www.escholarship.org/uc/item/2j2888zv (2014). 
5. Jelle, B. P., Breivik, C. \& Drolsum Røkenes, H. Building integrated photovoltaic products: A state-of-the-art review and future research opportunities. Sol. Energy Mater. Sol. Cells 100, 69-96 (2012).

6. Shrotriya, V. Organic photovoltaics: Polymer power. Nat. Photonics 3, 447-449 (2009).

7. Ababei, C., Yuvarajan, S. \& Schulz, D. L. Toward integrated PV panels and power electronics using printing technologies. Sol. Energy 84, 1111-1123 (2010).

8. Krishnan, S. et al. Novel dual gate high voltage TFT with variable doping slot. In 23rd International Conference on Microelectronics Proceedings. 1, 155-158 (2002).

9. Martin, R. A., Peng Kein Yap, Hack, M. \& Hsing, Tuan. Device design considerations of a novel high voltage amorphous silicon thin film transistor. In 1987 IEEE International Electron Devices Meeting. 440-443. Washington D. C. doi: 10.1109/IEDM.1987.191453 (1987)

10. Unagami, T. \& Tsuijiyama, B. High-voltage silicon thin film transistor on quartz. IEEE Electron Device Lett. 3, 3-4 (1982).

11. Xu, Y. Z. et al. High voltage polycrystalline thin-film transistor with variable doping slots in the offset region. Appl. Phys. Lett. 80, $2192(2002)$.

12. Dhyani, M. H. et al. Novel, 100V, Trench Super Junction high voltage TFTs using low temperature poly crystalline silicon. In 2009 IEEE International Electron Devices Meeting. 1-4. Baltimore, MD. doi: 10.1109/IEDM.2009.5424386 (2009).

13. Martin, R. A., Da Costa, V. M., Hack, M. \& Shaw, J. G. High-voltage amorphous silicon thin-film transistors. IEEE Trans. Electron Devices 40, 634-644 (1993).

14. Chow, E. M. et al. High voltage thin film transistors integrated with MEMS. Sensors Actuators A Phys. 130-131, 297-301 (2006).

15. Cheng, H.-C., Chang, F.-L., Lin, M.-J., Tsai, C. C. \& Liaw, C. W. Novel Low-Temperature Polycrystalline-Silicon Power Devices with Very-Low On-Resistance Using Excimer Laser-Crystallization. J. Electrochem. Soc. 151, G900 (2004).

16. Jamshidi-Roudbari, A., Kuo, P.-C. \& Hatalis, M. High Voltage, Moderate Current Thin Film Transistor for Actuator Applications. In ECS Transactions 11, 31-39 (2008).

17. Smith, M. A., Gowers, R. P., Shih, A. \& Akinwande, A. I. High-Voltage Organic Thin-Film Transistors on Flexible and Curved Surfaces. IEEE Trans. Electron Devices 62, 4213-4219 (2015).

18. Nomura, K. et al. Room-temperature fabrication of transparent flexible thin-film transistors using amorphous oxide semiconductors. Nature 432, 488-492 (2004).

19. Jeong, J., Lee, G. J., Kim, J., Kim, J.-H. \& Choi, B. High operating voltage application of transparent a-InGaZnO thin-film transistors. Semicond. Sci. Technol. 28, 025015 (2013).

20. Wang, L. Relationship between Intrinsic Breakdown Field and Bandgap of Materials. In 2006 25th International Conference on Microelectronics. Belgrade. 576-579, doi:10.1109/ICMEL.2006.1651032. (2006).

21. Hwang, W. S. et al. High-voltage field effect transistors with wide-bandgap $\beta-\mathrm{Ga}_{2} \mathrm{O}_{3}$ nanomembranes. Appl. Phys. Lett. 104, 203111 (2014).

22. Janotti, A. \& Van de Walle, C. G. Fundamentals of zinc oxide as a semiconductor. Reports Prog. Phys. 72, 126501 (2009).

23. Duan, Z., Du Pasquier, A., Lu, Y., Xu, Y. \& Garfunkel, E. Effects of Mg composition on open circuit voltage of $\mathrm{Cu}_{2} \mathrm{O}-\mathrm{Mg}_{\mathrm{x}} \mathrm{Zn}_{1-\mathrm{x}} \mathrm{O}$ heterojunction solar cells. Sol. Energy Mater. Sol. Cells 96, 292-297 (2012).

24. Law, M., Greene, L. E., Johnson, J. C., Saykally, R. \& Yang, P. D. Nanowire dye-sensitized solar cells. Nat. Mater. 4, 455-459 (2005).

25. Ku, C.-J. et al. Effects of $\mathrm{Mg}$ on the electrical characteristics and thermal stability of $\mathrm{Mg}_{\mathrm{x}} \mathrm{Zn}_{1-\mathrm{x}} \mathrm{O}$ thin film transistors. Appl. Phys. Lett. 98, 123511 (2011).

26. Ku, C.-J. et al. Improvement of Negative Bias Stress Stability in $\mathrm{Mg}_{0.03} \mathrm{Zn}_{0.97} \mathrm{O}$ Thin-Film Transistors. IEEE Electron Device Lett. 36, 914-916 (2015).

27. Huang, L. M. et al. Photovoltaic electrochromic device for solar cell module and self-powered smart glass applications. Sol. Energy Mater. Sol. Cells 99, 154-159 (2012).

28. Udatha, S. R. et al. Design Optimization of Thin-Film Transistors Based on a Metal-Substrate-Semiconductor Architecture for High DC Voltage Sensing. IEEE Trans. Electron Devices 63, 1696-1703 (2016).

29. Weisfield, R. L., Tuan, H. C., Fennell, L. \& Thompson, M. J. Amorphous Silicon Thin Film Transistor Array Technology: Applications in Printing and Document Scanning. MRS Proc. 95, 469 (1987)

30. Zhao, W., Waechter, D. \& Rowlands, J. a. Digital radiology using active matrix readout of amorphous selenium: Radiation hardness of cadmium selenide thin film transistors. Med. Phys. 25, 527 (1998).

31. Koike, K. et al. Radiation hardness of single-crystalline zinc oxide films. Phys. Status Solidi 9, 1577-1579 (2012).

32. Wie, C. R. Nonsaturating drain current characteristic in short-channel amorphous-silicon thin-film transistors. IEEE Trans. Electron Devices 57, 846-854 (2010).

33. Nomura, K., Kamiya, T., Ohta, H., Hirano, M. \& Hosono, H. Defect passivation and homogenization of amorphous oxide thin-film transistor by wet $\mathrm{O}_{2}$ annealing. Appl. Phys. Lett. 93, 192107 (2008).

34. Subramanian, M. A., Shannon, R. D., Chai, B. H. T., Abraham, M. M. \& Wintersgill, M. C. Dielectric constants of BeO, MgO, and $\mathrm{CaO}$ using the two-terminal method. Phys. Chem. Miner. 16, 741-746 (1989).

35. Posadas, a. et al. Epitaxial $\mathrm{MgO}$ as an alternative gate dielectric for SiC transistor applications. Appl. Phys. Lett. 92, 10-13 (2008).

\section{Acknowledgements}

This work was partially supported by the National Science Foundation under Grant No. CBET-1264508. The research was conducted (in part) at the Center for Functional Nanomaterials, Brookhaven National Laboratory, which was supported by the US Department of Energy, Office of Basic Energy Sciences, under Contract No. DE-SC0012704.

\section{Author Contributions}

The authors contributed to W.-C.H., C.-J.K., K.S. and Y.L. experimental design, W.-C.H., C.-J.K., R.L., S.-Y.W. and G.L. performance of experiments, W.-C.H., C.-J.K., R.L., S.A., P.R., M.L., K.S. and Y.L. data analysis and interpretation, W.-C.H., R.L. and Y.L.

\section{Additional Information}

Supplementary information accompanies this paper at http://www.nature.com/srep

Competing financial interests: The authors declare no competing financial interests.

How to cite this article: Hong, W.-C. et al. MgZnO High Voltage Thin Film Transistors on Glass for Inverters in Building Integrated Photovoltaics. Sci. Rep. 6, 34169; doi: 10.1038/srep34169 (2016). 
(c) (i) This work is licensed under a Creative Commons Attribution 4.0 International License. The images or other third party material in this article are included in the article's Creative Commons license, unless indicated otherwise in the credit line; if the material is not included under the Creative Commons license, users will need to obtain permission from the license holder to reproduce the material. To view a copy of this license, visit http://creativecommons.org/licenses/by/4.0/

(C) The Author(s) 2016 